\title{
IL GIOVANE JANKÉLÉVITCH TRA SIMMEL E BERGSON
}

\author{
Massimo Ferrari ${ }^{1}$
}

\begin{abstract}
RIASSUNTO: L'articolo ricostruisce l'interpretazione della filosofia della vita di Simmel che il giovane Jankélévitch propose in un articolo del 1925 apparso sulla Revue de Métaphysique et de Morale. Da queste pagine emerge non solo la profonda dimestichezza di Jankélévitch con Simmel, ma anche il tentativo compiuto da Jankélévitch per correggere l'elan vital di Bergson attraverso la concezione simmeliana della vita e della "tragedia della cultura".
\end{abstract}

PAROLE CHIAVE: Jankélévitch. Simmel. Bergson. Filosofia della vita. Filosofia francese.

Nel 1925 Vladimir Jankélévitvh pubblicava, sulle pagine della Revue de Métaphysique et de Morale, un lungo saggio in due puntate dedicato alla filosofia della vita di Georg Simmel. Il articolo del ventiduenne allievo dell'École Normale (ove ebbe tra i suoi maestri Henri Bergson e Léon Brunschvicg) è di grande finezza e merita ancora oggi di essere riletto. Esso documenta non solo la fase iniziale del pensiero di Jankélévitch, consentendo di cogliere l'avvio del percorso che lo porterà al più noto e importante libro su Bergson del 1931, ma costituisce al tempo stesso una preziosa testimonianza della ricezione francese di Simmel: una vicenda di singolare interesse, in particolare per quanto riguarda proprio il nesso Bergson-Simmel e, per un altro verso, la fortuna di Bergson nella cultura filosofica tedesca tra la belle époque e il drammatico periodo postbellico (FITZI, 2002; ZANFI, 2013).

Il giovane Jankélévitch aveva già pubblicato, sulla Revue philosophique de la France et de l'étranger del 1924, un articolo su Jean-Marie Guyau e Bergson che lo stesso Bergson aveva dapprima letto in manoscritto, per poi

\footnotetext{
${ }^{1}$ Massimo Ferrari è Professore ordinario di Storia della filosofia presso l’Università di Torino. Si occupa di neokantismo, fenomenologia, pragmatismo, empirismo logico, storia della filosofia della scienza, nonché del pensiero francese tra Ottocento e Novecento e della storia della filosofia italiana. Tra le sue pubblicazioni Categorie e a priori (Bologna, 2003), Ernst Cassirer. Stationen einer philosophischen Biographie (Hamburg, 2003), Non solo idealismo (Firenze, 2006). Collabora a numerose riviste italiane e straniere. E' stato visiting professor all'Università della Sorbona e attualmente ha in preparazione la biografia intellettuale de Moritz Schlick. massimo.ferrari@unito.it
} 
elogiarlo nella versione a stampa - in una lettera a Jankélévitch del 12 maggio di quell'anno - definendolo "penetrante». Aggiungeva Bergson: «[...] questo primo lavoro fa presagire altre opere che saranno un contributo importante al pensiero filosofico» (BERGSON, 2002, p. 1090). In effetti, in quel confronto tra Guyau e Bergson nel quadro di una spiccata attenzione per la filosofia della vita e i suoi diversi orientamenti, Jankélévitch mostrava la sua profonda vicinanza a Bergson e il suo interesse per la discussione sulla Lebensphilosophie fiorita nei primi anni del Novecento, alimentata, più di recente, dal libro invero piuttosto polemico di Heinrich Rickert Die Philosophie des Lebens: un testo che Jankélévitch espressamente citava giudicandolo «ammirevole» (JANKÉLÉVITCH, 1994, p. 14 n. 1; RICKERT, 1920).

Il saggio su Guyau e Bergson è - a sua volta - un lavoro non meno «ammirevole». Jankélévitch conduce un minuzioso raffronto tra due filosofie della vita che sembrano convergere in più punti, ma che muovono $\mathrm{da}$ prospettive diverse: mentre per Bergson non si tratta di elevare la materia all'altezza della vita, bensì di epurare la vita da ogni elemento materiale, Guyau non sembra andare oltre una concezione ancora meccanicistica della natura (JANKÉLÉVITCH, 1994, p. 21). Non sfuggiva d'altro canto a Jankélévitch la centralità, in Guyau, della rifondazione della morale scientifica basata sul ruolo del sentimento, dello slancio della vita verso l'alter che deve però rafforzare "la vera "autonomia"», mirando - a differenza di Kant all' «originalità individuale» e non già all' «uniformità universale» (GUYAU, 1903, p. 166). Era questa l'origine del disaccordo «essenziale» con Bergson, dal momento che Guyau, osservava Jankélévitch, in realtà è "un moralista» (JANKÉLÉVITCH, 1994, p. 33). A partire di qui il raffronto procedeva sui temi della durata, dell'intuizione, dell'estetica; e l'accento cadeva ripetutamente sul perdurante intellettualismo di Guyau, pur riconoscendogli il merito di aver formulato, ben prima di Bergson e di Simmel, «[...] i principi del metodo dell'intuizione» (JANKÉLÉVITCH, 1994, p. 57). Il bilancio del saggio di Jankélévitch si faceva complesso: oltre a Simmel, compariva anche Nietzsche e - su tutto - l'orizzonte di «una autentica filosofia della vita», che non assumeva però il volto esclusivo di Bergson. Anzi, era se mai Simmel a indicare la strada da seguire: perché, in parte non diversamente da Guyau, aveva saputo fondere «felicemente» l'intuizionismo bergsoniano con la considerazione dell' «ambiente sociale» in cui «l'io vivente» va oltre l'attimo fuggente del vivere. La durata vitale deve anche «immobilizzarsi»: l'intuizione della vita - annotava Jankélévitch con Simmel - è fatta al tempo stesso della conoscenza dei nostri limiti e del nostro sentimento del trascenderli; eliminare 
dunque le forme statiche e stereotipate di un concettualismo senza futuro è parte essenziale dei "compiti che ricadono ora sui giovani filosofi della vita» (JANKÉLÉVITCH, 1994, p. 62).

Nel saggio del 1924 Simmel non era dunque una presenza casuale, e anzi costituiva un punto di riferimento indispensabile per Jankélévitch - anche perché Simmel, e il confronto tra Simmel e Bergson, avevano rappresentato un episodio tutt'altro che marginale nelle 'cronache di filosofia' in Francia prima del conflitto mondiale. Il filosofo tedesco era noto nella cultura francese fin de siècle grazie alla traduzione di alcuni contributi sociologici (il primo di essi, uscito nel 1894, presentava una parte del libro sulla Soziale Differenzierung). I contatti si erano intensificati negli anni seguenti, coinvolgendo figure come Celestin Bouglé ed Emile Durkheim, ma raggiungendo anche il fronte filosofico grazie alla mediazione della Revue de Métaphysique et de Morale e all'ambiente legato all'organizzazione del I Congresso Internazionale di Filosofia di Parigi nel 1900, a cui Simmel partecipò con una relazione intitolata De la religion au point de vue de la théorie de la connaissance (SIMMEL 2002, p. 117-128; FITZI 2006, p. 19-32). Tuttavia il Simmel filosofo avrebbe conosciuto una più larga circolazione soprattutto a partire dal 1912, quando uscirono presso l'editore Alcan di Parigi i Mélanges de philosophie relativiste. Contribution à la culture philosophique: una raccolta di saggi che spaziavano da Schopenhauer a Nietzsche, da Kant a Rodin, dalle famose riflessioni sulla «metafisica della morte» ai temi caratteristici del Simmel filosofo della cultura (l'arte, l'esperienza religiosa, la conoscenza storica, la natura della filosofia, l'analisi della modernità). Lo stesso Simmel scrisse una prefazione al volume tradotto da Alix Guillain, per sottolineare in qual senso il suo lavoro di «[...] comprensione della vita» fosse guidato, più che dalla filosofia in senso tradizionale, da un' «attitudine spirituale» definibile come «cultura filosofica» (SIMMEL, 2002, p. 139). Insisteva Simmel:

[...] per la cultura filosofica è come per la cultura religiosa, che non consiste nell'accettazione di un dogma, bensì nella comprensione della vita, considerando che si tratta di conformare la propria esistenza avendo costantemente presente allo spirito l'eterna sorte dell'anima; oppure si potrebbe fare ancora un paragone con la cultura artistica, che non consiste della somma delle singole opere d'arte, bensì nella circostanza in base alla quale i contenuti dell'esistenza si formano per noi in base alle norme artistiche. 
Ma non si dà mai - concludeva Simmel - una forma cristallizzata che venga riempita da un pensiero fondato su un solo principio immobile: esso piuttosto la colma con «[...] un mosaico composto dai frammenti di un pensiero dello stesso genere» (SIMMEL, 2002, p. 141).

Già prima della pubblicazione dei Mélanges era iniziato il sodalizio tra Simmel e Bergson, che pur non essendo ancora in contatto personale avevano avuto numerose occasioni di familiarizzarsi con le rispettive posizioni filosofiche (FITZI, 2002, p. 53). L'8 dicembre 1908 Bergson scriveva infine a Simmel per ringraziarlo di avergli inviato la terza edizione dei Probleme der Geschichtsphilophie, la cui lettura aveva appena intrapreso con grande interesse. Ma non era un omaggio rituale: Bergson osservava che, nonostante le loro ricerche filosofiche vertessero su oggetti diversi, era possibile scorgere «una certa affinità», d'altronde riconosciuta dallo stesso Simmel. "Una delle ragioni di questa affinità - soggiungeva Bergson - può essere che ci troviamo d'accordo nel distinguere, nel fatto, ciò che ci è dato direttamente dall'esperienza e ciò che vi aggiunge lo spirito in vista di esigenze pratiche» (BERGSON, 2002, p. 231-232). Per quanto sia andata perduta la successiva corrispondenza tra Simmel e Bergson, non è difficile dunque comprendere per quali ragioni il loro rapporto dovesse farsi più stretto negli anni seguenti, tanto più che già nel 1909 sarebbero apparse - grazie anche all'iniziativa di Simmel - le traduzioni tedesche dell'Evolution créatrice e dell'Introduction à la métaphysique. La rete dei rapporti (piuttosto fitta, al di là di Simmel) e la presenza crescente di Bergson nell'ambiente filosofico tedesco portarono così a una sorta di incrocio tra l'élan vital e la filosofia della vita in Germania, e più in generale all'intensificarsi delle relazioni tra i filosofi tedeschi e quello che Isaak Benrubi chiamerà, nel 1914, il «risveglio filosofico» della Francia (FITZI, 2002, p. 217-237; BENRUBI, 1914).

Ma il documento forse più rilevante è costituito dalle pagine che Simmel consacrerà a Bergson in un articolo del 1914: due filosofie della vita a confronto, tra l'incessante élan vital del filosofo francese e la «tragedia» della cultura - della frattura tra la vita e le forme - messa a fuoco da Simmel. Bergson compariva qui come l'alfiere di quel rovesciamento del meccanicismo ottocentesco verso la vita che Simmel non poteva che sottoscrivere. La vita è un flusso incessante, una continua emergenza del nuovo irriducibile al nesso tra causa ed effetto, ma piuttosto consegnato a un "movimento creatore» (SIMMEL, 2000, p. 58). Simmel rilevava tuttavia la vicinanza di Bergson al pragmatismo (e questo, in realtà, era già uno spunto critico), ma soprattutto 
teneva a sottolineare come nella prospettiva di Bergson mancasse un "ponte» tra le premesse soggettive e le conseguenze oggettive del nostro agire, ciò che in ultima analisi lo portava a non riconoscere l'aspetto tragico della vita. "Anche solo per esistere - scriveva Simmel - la vita deve trasformarsi nella non-vita» (SIMMEL, 2000, p. 63). Le vie di Simmel e Bergson sembravano qui separarsi: agli occhi di Simmel, Bergson non teneva adeguatamente conto di come la vita dell'uomo oscilli tra il fluire e il consolidarsi, istituendo un contrasto tra ciò che si sviluppa senza sosta e ciò che si solidifica nelle forme alimentate dalla vita. «Forse - concludeva Simmel - la filosofia farà il suo prossimo passo conquistando un concetto di vita che sia davvero al di là di queste opposizioni»: all'altezza di un'unità metafisica della vita che sciolga l'assolutezza del contrasto tra i suoi due poli (SIMMEL, 2000, p. 69).

Alla vigilia del conflitto mondiale, dopo un dialogo diretto e indiretto durato diversi anni, la posizione di Simmel di fronte a Bergson sembrava così delinearsi definitivamente. Proprio nel 1914, del resto, Albert Mamelet raccoglieva in volume (con un' introduzione di Victor Delbos) un saggio apparso in più puntate sulla Revue de Métaphysique et de Morale l'anno precedente. Il lavoro di Mamelet (che Jankélévitch apprezzerà come «un'esposizione fedele e intelligente» [JANKÉLÉVITCH, 2013, p. 21]) si incentrava sul «relativismo» di Simmel, le cui origini venivano individuate giustamente nella prima grande opera di Simmel, la Einleitung in die Moralwissenschaft che già faceva presagire la successiva filosofia della vita (MAMELET, 1914, p. 58). Per quanto ancora non potesse conoscere la summa della Lebensphilosophie di Simmel (la Lebensanschauung uscirà solo nel 1918) Mamelet seguiva con acume lo sviluppo del pensiero di Simmel e insisteva in maniera persuasiva sull'importanza, per il «relativismo» simmeliano, della sua imponente Filosofia del denaro (uscita nel 1900) e della originale rilettura del criticismo documentata dalle lezioni berlinesi confluite nella monografia su Kant pubblicata in prima edizione nel 1904 (MAMELET, 1914, p. 76).

Con il libro di Mamelet si chiude sostanzialmente - all'insegna della categoria del «relativismo» - la prima ricezione di Simmel nella filosofia francese. Lo scoppio del conflitto mondiale in quello stesso 1914 interruppe del resto il dialogo culturale e filosofico tra Francia e Germania, che pure era stato lungamente presente sulla scena europea e aveva rappresentato per molti eminenti filosofi francesi (si pensi ad esempio a Émile Boutroux) un aspetto cruciale della loro formazione e della loro appartenenza alla comunità filosofica internazionale (FERRARI, 2014). La morte di Simmel nel 1918 e il 
clima arroventato del primo dopoguerra a seguito della pace di Versailles non favorirono certo la ripresa di quel dialogo che aveva caratterizzato il 'mondo di ieri' inabissatosi con l'attentato di Sarajevo. Lo testimonia d'altra parte il caso emblematico dell'ostilità con cui venne accolta in Germania la decisone di Albert Einstein di partecipare, nel 1922, a un incontro promosso a Parigi dalla Societé Française de Philosophie per discutere della teoria della relatività: un celebre dibattito in cui Bergson fu uno dei protagonisti e che in qualche modo riallacciò, o volle seppur debolmente riallacciare, il filo spezzato dello scambio intellettuale tra le due sponde del Reno.

E' in questo contesto che Jankélévitch, nel 1925, tentò di ritessere un altro legame interrottosi con la guerra rileggendo le opere del 'relativista' Simmel. Il punto nevralgico del saggio di Jankélévitch è il ripetuto confronto con Bergson, che mette in luce (riferendosi a Lebensanschauung) l'«ispirazione» bergsoniana di Simmel, «invisibile» eppure «presente» in tutte le sue pagine ultime. "Bergson - rileva Jankélévitch - non è mai nominato in Intuizione della vita, ma l'ispirazione bergsoniana, invisibile e presente, penetra, attraversa, permea tutto il volume» (JANKÉLÉVITCH, 2013, p. 41). Jankélévitch rintraccia questa "presenza» con un'acuta sensibilità bergsoniana: legge Simmel attraverso Bergson e ritrova in Simmel i problemi di Bergson. Egli riscontra anche una matrice comune nell'affrontare l'eredità di Kant, accolta da entrambi in una prospettiva peculiare: «Bergson, non meno di Simmel, non contende a Kant il merito di aver spostato l'attenzione filosofica dai concetti, che sono cose, alle leggi, che sono relazioni» (JANKÉLÉVITCH, 2013, p. 27). Naturalmente, non sfugge a Jankélévitch il diverso modo di collocarsi nei confronti del criticismo kantiano e lo sottolinea tuttavia in termini che non sono (come invece lo sarà in seguito) di opposizione radicale: «Simmel è erede della tradizione criticista, ma come Bergson, forse ancora più di lui, ha subito contemporaneamente l'influsso dell'evoluzionismo, del pragmatismo e del "sociologismo" (JANKÉLÉVITCH, 2013, p. 28). In realtà, Jankélévitch sarà sempre meno disposto a legare Bergson alla "tradizione criticista» e anzi avrà modo di sottolineare, molti anni più tardi, come nella sua costante avversione per Charles Renouvier (seppure mai citato espressamente) Bergson mirasse anche al criticismo: "Kant est son adversaire de toujours; son frappe à la tête: de l'Essai sur les données immédiates aux Deux sources de la morale et de la religion, Bergson est resté fidèle à lui-même dans cette animadversion» (JANKÉLÉVITCH, 1965, p. vii). 
Non è tuttavia su questo punto più specifico, per quanto rilevante, che Jankélévitch intende porre l'accento. La sua ammirazione per Simmel, innanzi tutto, si rende manifesta nella critica al libro di Rickert che egli aveva ricordato l'anno precedente: perché negare la forza espansiva della vita, la sua inarrestabile tendenza a superarsi e a ritornare a sé piuttosto che «abolirsi in un'eternità inumana?». Non è forse proprio questo il "grandissimo merito» di Simmel? Scrive Jankélévitch: «Rickert rimprovera a Simmel di non aver imposto una forma statica, fosse anche la forma del cambiamento. Io ritengo che proprio qui stia il suo grandissimo merito, la sua incomparabile originalità» (JANKÉLÉVITCH, 2013, p. 75). Nella concezione simmeliana della vita che non si arresta al suo fluire, ma accresce se stessa ("più vita») e si oggettiva in forme che la superano ("più-che-vita»), la irrigidiscono pur dovendo poi nel loro constante mutare ritornare alla vita, Jankélévitch scorge «la grande riforma» di Simmel (JANKÉLÉVITCH, 2013, p. 76). E' il Simmel del famoso saggio del 1911 sulla Tragedia della cultura, è il Simmel della Lebensanchauung del 1918 su cui si chiude la sua vita filosofica. Ed è a questo Simmel che Jankélévitch guarda con profonda simpatia intellettuale: un momento centrale della filosofia simmeliana che gli sembra vicina - persino più vicina di quanto non lo sia quella di Bergson - in virtù del suo aspetto tragico, della sua accettazione che "[...] il male, che ci uccide, è una necessità pressoché ineluttabile» (JANKÉLÉVITCH, 2013, p. 76).

Non per nulla Jankélévitch sottolinea con forza la diversità tra la concezione 'eraclitea' della vita formulata da Bergson e il "più che vita» di Simmel, ciò che rappresenta a suo avviso una prospettiva «totalmente antibergsoniana» essendo la vita che trascende se stessa non più autentica vita (JANKÉLÉVITCH, 2013, p. 70). In realtà, scrive Jankélévitch nell'ultima pagina del suo saggio, Simmel è il filosofo che coglie il carattere febbrile e vibrante della vita moderna, consapevole con pessimismo che la «tragedia della cultura» non può essere risolta e che non si tratta del semplice «irrazionalismo» denunciato da «un vecchio idealista come Windelband». E conclude, con parole che esprimono bene tutta la sua "attenzione» e tutta la sua «simpatia» per Simmel: «In questo pensiero mobile e perpetuamente inquieto, c'è un che di febbrile, di angosciato e di vibrante, specificamente moderno. Georg Simmel non è un ottimista, e la sua tragedia della cultura non può certo prometterci il riposo e la quiete: come Pascal, Georg Simmel approverebbe volentieri coloro che "cercano gemendo" (JANKÉLÉVITCH, 2013, p. 82). 
Nella «tragedia» simmeliana si può dunque individuare una fonte importante della filosofia di Jankélévitch, anche nei suoi sviluppi più tardi all'interno del «momento» esistenzialistico della filosofia francese: all'insegna di una dualità irriducibile nella nostra esistenza, tra un polo che tenacemente resiste e un altro, contrapposto, che è evanescente e fuggitivo (WORMS, 2009, p. 402-403). Non sembra peraltro casuale che il nome di Simmel continui a circolare nelle pagine di Jankélévitch, in termini non diversi da quelli espressi nel suo saggio del 1925. In un passo della Mauvaise conscience del 1966, ad esempio, Simmel ricompare nel contesto di una meditazione sulla «trappola» che la volontà tende all'uomo, dividendolo tra l'inclinazione ad astenersi e la tendenza a farsi coinvolgere dalle proprie opere. E’ il dramma dell'esistenza, a cui Simmel «[...] conferisce un andamento appassionato e molto attuale chiamandola "tragedia della cultura"; la vita cerca delle forme e uno stile per esprimersi; ma le forme e gli stili si ritorcono contro la vita; così la religione soccombe ai dogmi, la morale ai "gesti" e il diritto al formalismo dei codici; i ricordi costituiti, infine, uccidono la memoria costituente [...] $\mathrm{La}$ "tragedia della cultura” è, per forza di cose, una tragedia dell'espressione» (JANKÉLÉVITCH, 2000, p. 153).

Resterebbe da documentare, a questo punto, quanto Simmel sia stato presente nel grande libro su Bergson del 1931, preparato dal saggio su Bergson e la biologia che Bergson stesso considerò nuovamente con particolare favore. «Lei - scriveva Bergson a Jankélévitch il 27 maggio 1929 - ha fatto molto onore ai miei lavori e gli ha reso un vero sevizio mostrando come essi abbiano potuto anticipare alcuni risultati della scienza positiva» (BERGSON, 2002, p. 1301). Ma Bergsonisme et biologie (uscito nel 1929 sulla Revue de Métaphisique et de Morale) toccava un punto specifico del pensiero di Bergson, nel tentativo di mostrare come la biologia contemporanea - in particolare la neurobiologia di Monakow e Mourgue - mettesse in luce spiccate affinità con l'Evolution créatrice (JANKÉLÉVITCH, 1994, p. 64-76). Il libro del 1931 era invece non solo un libro su Bergson, ma un libro con Bergson, che lo stesso Bergson - in una lettera a Jankélévitch del gennaio 1931 - giudicava «uno studio singolarmente approfondito e originale» (BERGSON, 2002, p. 1340). Sono in effetti pagine mirabili, in cui si avvertono echi molteplici della filosofia contemporanea (e basti fare il nome di Brunschvicg), in un'atmosfera già esistenzialistica e sensibile ai momenti tragici della vita umana, che apparivano quanto meno appannati in Bergson e che invece proprio Simmel aveva compreso. Simmel: il filosofo capace di cogliere con estrema finezza - riconosce a un certo punto Jankélévitch, riferendosi a Der 
Konflikt der modernen Kultur - che solo tramite la vita si può penetrare, si può comprendere e intuire la vita stessa (JANKÉLÉVITCH, 1959, p. 74). Né sarebbe difficile, ripercorrendo dettagliatamente le pagine di quel libro, appurare quanto Simmel (esplicitamente o implicitamente) sia ancora e sempre presente a Jankélévitch, quasi a delineare un 'bergsonismo simmeliano' che rappresenta un unicum nella filosofia francese (e non solo francese) tra le due guerre mondiali.

Certo è, in definitiva, che Jankélévitch fu veramente il «mediatore» tra Simmel e la filosofia di Bergson, in un momento di trasformazione profonda del pensiero europeo del Novecento (ZANFI, 2013, p. 117-132). Il contributo di Jankélévitch al «bergsonismo» (DELEUZE, 1966) apre del resto una prospettiva importante per capire il pensiero di Jankélévitch, che probabilmente deve molti di più di quanto non si ritenga comunemente a quei primi lavori degli anni Venti, in cui le due 'voci' dell' élan bergsoniano e della 'tragedia della cultura' simmeliana si erano unite nell'intonare il canto della vita, della sua fenomenologia colta in quel «non so che» nel quale è riposto il movimento profondo della coscienza dell'uomo, il mistero della sua «ipseità».

FERRARI, Massimo. The young Jankélévitch between Simmel and Bergson. Trans/Form/ Ação, Marília, v. 37, p. 209-218, 2014. Edição Especial.

\begin{abstract}
This paper aims at showing the interpretation of Simmel's philosophy of life that the young Jankélévitch proposed in a study published in 1925 in the Revue de Métaphysique et de Morale. This essay not only highlights Jankélévitch's deep acquaintance with Simmel, but also his attempt to correct Bergson's élan vital through Simmel's conception of both life and "tragedy of culture".
\end{abstract}

KEYWORDS: Jankélévitch. Simmel. Bergson. Philosophy of Life. French philosophy.

\title{
BibLIOGRAFIA
}

BENRUBI, Isaak. Emile Boutroux und das philosophische Erwachen der Gegenwart. Internationale Monatsschrift für Wissenschaft, Kunst und Technik, v. 8, p. 929-952, 1914.

BERGSON, Henri. Correspondances. Textes publiés et annotés par André Robinet. Paris: Presses Universitaires de France, 2002. 
BARTHÉLEMY-MADAULE, Madeleine. Bergson adversaire de Kant. Paris: Presses Universitaires de France, 1966.

DELEUZE, Gilles. Le bergsonisme, Paris: Presses Universitaires de France, 1966.

FERRARI, Massimo. Émile Boutroux (di prossima pubblicazione).

FITZI, Gregor. Soziale Erfahrung und Lebensphilosophie. Georg Simmels Beziehung zu Henri Bergson. Konstanz: Verlagsgesellschaft, 2002.

GUYAU, Jean-Marie. Esquisse d'une morale sans obligations et sanctions. Paris: Alcan, $1903^{3}$. JANKÉLÉVITCH, Vladimir. Henri Bergson. Paris: Presses Universitaires de France, 1959. . "Préface". In: BARTHÉLEMY-MADAULE, Madeleine. Bergson adversaire de Kant. Paris: Presses Universitaires de France, 1966. . Premières et dernières pages. Paris: Éditions du Seuil, 1994. - La cattiva coscienza. A cura di D. Discipio. Bari: Dedalo, 2000. . Georg Simmel filosofo della vita. A cura di L. Boella. Milano: Mimesis, 2013. MAMELET, Albert. Le relativisme philosophique chez Georg Simmel. Paris: Alcan, 1914. RICKERT, Heinrich. Die Philosophie des Lebens. Darstellung und Kritik der philosophischen Modeströmungen unserer Zeit. Tübingen: Mohr, 1920.

SIMMEL, Georg. Aufsätze und Abhandlungen 1909-1918. Frankfurt am Main: Suhrkamp, 2000. v. 2. (Gesamtausgabe, v. 13)

Französisch-und italienischsprachige Veröffentlichungen. Mélanges de philosophie relativiste. Frankfurt am Main: Suhrkamp, 2002. (Gesamtausgabe, v. 19)

WORMS, Frédéric. La philosophie en France au XXe siècle: moments. Paris: Gallimard, 2009.

ZANFI, Cristina. Bergson e la filosofia tedesca 1907-1932. Macerata: Quodlibet, 2013. 